\title{
Theoretical evaluation of ultrasonic velocities in binary liquid mixtures at different temperatures
}

\author{
M. Durga Bhavani, A. Ratnakar*, Ch. Kavitha \\ Department of Chemistry, V.R.Siddhartha Engineering College, Vijayawada, Andhra Pradesh, India \\ *E-mail address: drabbineniratnakar@gmail.com
}

\begin{abstract}
Ultrasonic velocities calculated from various theories and relations like Nomoto's relation, Van dael ideal mixing relation, Impedance relation, Rao's specific velocity relation and Jungie's theory are compared with experimental values in binary liquid mixtures o-anisidine with o-cresol at temperatures $303.15,308.15,313.15$ and $318.15 \mathrm{~K}$ over the entire mole fraction range. The relative applicability of these theories to the present system is checked and discussed. A good agreement is observed between experimental and theoretical values. The results are explained in the light of molecular interactions occuring in these mixtures.
\end{abstract}

Keywords: Binary liquid mixtures; o-anisidene, o-cresol; Nomoto; ultrasonic velocity

\section{INTRODUCTION}

Results of theoretical evaluation of ultrasonic velocities are used for the better understanding of molecular arrangements in liquid mixtures. In assessing the nature of molecular interactions and investigating the physico-chemical behaviour, ultrasonic study of liquid mixtures gained more importance during the last few decades. Several researchers [1-4] carried out ultrasonic investigations on binary and ternary liquid mixtures and compared the experimental values with theoretical relations [5-9] of Nomoto, Van Dael and Vangeel, Impedance dependence, Rao's specific velocity and Junjie's equations and the results are explained in terms of molecular interactions. o-anisidine is mainly used in the manufacture of dyes for tattooing and coloration of paper. Cresols are used to dissolve other chemicals, as disinfectants and deodorizers, and to make specific chemicals that kill insect pests. Ultrasonic velocities calculated in binary liquid mixture o-anisidine with o-cresol using the above relations are compared with the experimental values at temperatures $303.15,308.15,313.15$ and $318.15 \mathrm{~K}$ for the entire mole fraction range. 


\section{EXPERIMENTAL}

The chemicals used in this work are obtained from Loba (o-anisidine, purity $98 \%$ ) and SDFCL (o-cresol, purity $99 \%$ ) and were used as such without further purification. The purity of the samples was checked by comparing the experimental values of density with the values reported in literature [10]. The mixtures of required proportions are prepared by using Job's method of continuous variation and are preserved in well-stoppared conical flasks. The flasks are left free to allow them to attain thermal equilibrium after they are prepared.

Using ultrasonic interferometer (Mittal enterprises, India; Model: F-80X) ultrasonic velocities were measured. It consists of a high frequency generator and a measuring cell and the measurements were made at a fixed frequency of $3 \mathrm{MHz}$. The calibration of the equipment was done by measuring the velocity in water and benzene, and the results were compared with the literature values [11]. The ultrasonic velocity has an accuracy of $\pm 0.5 \%$. Temperature was controlled by circulating water around the liquid cell from thermostatically controlled constant temperature water bath. Using specific gravity bottle, the densities of pure liquids and liquid mixtures were measured. Weights were measured with an electronic balance (Shimadzu AUY220, Japan) capable of measuring up to 0.1mg. An average of 4-5 measurements was taken for each sample.

\section{RESULTS AND DISCUSSION}

Theoretical values of ultrasonic velocities were calculated using different theories and empirical relations. Comparison of theoretical values of ultrasonic velocities with those obtained experimentally in the present binary liquid mixtures is expected to reveal the nature of interaction between the component molecules in the mixture. Such theoretical study is useful in building the comprehensive theoretical model for the liquid mixtures. Theoretical values of ultrasonic velocities in the mixtures o-anisidine + o-cresol at different mole fractions of o-anisidine for different temperatures were calculated using the following theories and relations:

Nomoto relation for ultrasonic velocity in binary liquid mixtures,

$$
\mathrm{U}_{\mathrm{N}}=\left[\left(\mathrm{x}_{1} \mathrm{R}_{1}+\mathrm{x}_{2} \mathrm{R}_{2}\right) /\left(\mathrm{x}_{1} \mathrm{~V}_{1}+\mathrm{x}_{2} \mathrm{~V}_{2}\right)\right]^{3}
$$

Where $\mathrm{R}$ is molar sound velocity, $\mathrm{x}_{1}$ and $\mathrm{x}_{2}$ are the mole fractions of $1^{\text {st }}$ and $2^{\text {nd }}$ components of the liquid mixture and $\mathrm{V}$ is molar volume.

Van Dael and Vangeel Ideal mixing relation,

$$
\mathrm{U}_{\mathrm{imx}}=\left[\left(\mathrm{x}_{1} / \mathrm{M}_{1} \mathrm{U}^{2}{ }_{1}+\mathrm{x}_{2} / \mathrm{M}_{2} \mathrm{U}^{2}{ }_{2}\right)\left(\mathrm{x}_{1} \mathrm{M}_{1}+\mathrm{x}_{2} \mathrm{M}_{2}\right)\right]^{-1 / 2}
$$

where $U_{\mathrm{imx}}$ is the ideal mixing ultrasonic velocity in liquid mixture. $\mathrm{U}_{1}$ and $\mathrm{U}_{2}$ are ultrasonic velocities of the individual compounds.

Impedance dependent relation,

$$
\mathrm{U}_{\mathrm{Im}}=\Sigma \mathrm{x}_{\mathrm{i}} \mathrm{Z}_{\mathrm{i}} / \Sigma \mathrm{x}_{\mathrm{i}} \rho_{\mathrm{i}}
$$


where $\mathrm{x}_{\mathrm{i}}$ is the mole fraction, $\rho_{\mathrm{i}}$ the density of the mixture and $\mathrm{Z}_{\mathrm{i}}$ is the acoustic impedance. Rao's specific velocity,

$$
\mathrm{U}_{\mathrm{R}}=\left(\Sigma \mathrm{x}_{\mathrm{i}} \mathrm{r}_{\mathrm{i}} \rho_{\mathrm{i}}\right)^{3}
$$

where $x_{i}$ is the mole fraction, $\rho_{i}$ the density of the mixture and $r_{i}$ is the Rao's specific sound velocity.

Jungie equation,

$$
\mathrm{U}_{\mathrm{J}}=\left(\mathrm{x}_{1} \mathrm{M}_{1} / \rho_{1}+\mathrm{x}_{2} \mathrm{M}_{2} / \rho_{2}\right) /\left[\left\{\mathrm{x}_{1} \mathrm{M}_{1}+\mathrm{x}_{2} \mathrm{M}_{2}\right\}^{1 / 2} \mathbf{x}\left\{\mathrm{x}_{1} \mathrm{M}_{1} / \rho_{1} \mathrm{U}^{2}{ }_{1}+\mathrm{x}_{2} \mathrm{M}_{2} / \rho_{2} \mathrm{U}^{2}{ }_{2}\right\}\right]^{1 / 2}
$$

where $M_{1}, M_{2}$ are molecular weights of constituent components, $\rho_{1}$ and $\rho_{2}$ are the densities of constituent components. The theoretical evaluation of sound velocity based on different models in liquid mixtures has been used to correlate with the experimental findings. The theoretical values of ultrasonic velocities calculated by using the Equations (1-5) along with the experimental values for the binary mixtures at temperatures of $303.15,308.15,313.15$ and $318.15 \mathrm{~K}$ are given in Table 1 . The validity of these theories is checked by percentage deviation for the mixtures at all the temperatures and is given in Tables 2. It can be observed from Table 1 that the theoretical values of ultrasonic velocity calculated by using various theories show deviation from experimental values. The limitations and approximation incorporated in these theories are responsible for the deviations of theoretical values from experimental values. In Nomoto's theory, it is supposed that the volume does not change on mixing. But on mixing two liquids, the interaction between the molecules of the two liquids takes place because of the presence of various types of forces such as dispersive forces, charge transfer, hydrogen bonding, dipole-dipole and dipole-induced dipole interactions.

Thus, the observed deviation of theoretical values of velocity from the experimental values shows that the molecular interactions are taking place [12,13] between the unlike molecules in the liquid mixture. From Table 2, more deviations are observed in case of Nomoto theory and less deviation are observed in case of Van dael ideal mixing relation. On increasing temperature, it was observed that the ultrasonic velocity values decrease in the liquid mixtures chosen. This is probably due to the fact that the thermal energy activates the molecule, which would increase the rate of association of unlike molecules.

Table 1. Experimental and theoretical values of velocities in o-anisidine + o- cresol system at different

\begin{tabular}{|c|c|c|c|c|c|c|}
\hline $\mathrm{x}_{1}$ & $\mathrm{U}_{\exp }$ & $\mathrm{U}_{\mathrm{N}}$ & $\mathrm{U}_{\text {imx }}$ & $\mathrm{U}_{\mathrm{Im}}$ & $\mathrm{U}_{\mathrm{R}}$ & $\mathrm{U}_{\mathrm{J}}$ \\
\hline \multicolumn{7}{|c|}{$303.15 \mathrm{~K}$} \\
\hline 0.0000 & 1485.26 & 1485.26 & 1485.26 & 1485.26 & 1485.26 & 1459.09 \\
\hline 0.0929 & 1492.10 & 1496.28 & 1494.85 & 1496.18 & 1495.49 & 1465.52 \\
\hline 0.1872 & 1500.00 & 1507.35 & 1504.77 & 1507.18 & 1505.93 & 1472.13 \\
\hline 0.2831 & 1509.47 & 1518.47 & 1515.02 & 1518.25 & 1516.58 & 1478.94 \\
\hline 0.3805 & 1521.42 & 1529.65 & 1525.62 & 1529.39 & 1527.47 & 1485.95 \\
\hline 0.4795 & 1527.36 & 1540.89 & 1536.60 & 1540.61 & 1538.58 & 1493.16 \\
\hline 0.5802 & 1542.00 & 1552.18 & 1547.97 & 1551.91 & 1549.93 & 1500.59 \\
\hline
\end{tabular}




\begin{tabular}{|c|c|c|c|c|c|c|}
\hline 0.6825 & 1552.46 & 1563.52 & 1559.76 & 1563.29 & 1561.53 & 1508.23 \\
\hline 0.7865 & 1564.21 & 1574.92 & 1571.99 & 1574.74 & 1573.39 & 1516.10 \\
\hline 0.8924 & 1579.47 & 1586.38 & 1584.69 & 1586.28 & 1585.50 & 1524.21 \\
\hline 1.0000 & 1597.89 & 1597.89 & 1597.89 & 1597.89 & 1597.89 & 1532.56 \\
\hline \multicolumn{7}{|c|}{$308.15 \mathrm{~K}$} \\
\hline 0.0000 & 1466.84 & 1466.84 & 1466.84 & 1466.84 & 1466.84 & 1444.62 \\
\hline 0.0929 & 1474.52 & 1477.51 & 1476.12 & 1477.39 & 1476.74 & 1450.92 \\
\hline 0.1872 & 1482.36 & 1488.24 & 1485.70 & 1488.01 & 1486.84 & 1457.40 \\
\hline 0.2831 & 1491.63 & 1499.01 & 1495.62 & 1498.71 & 1497.15 & 1464.06 \\
\hline 0.3805 & 1503.05 & 1509.83 & 1505.87 & 1509.49 & 1507.68 & 1470.91 \\
\hline 0.4795 & 1509.36 & 1520.70 & 1516.49 & 1520.34 & 1518.43 & 1477.95 \\
\hline 0.5802 & 1522.84 & 1531.62 & 1527.49 & 1531.27 & 1529.41 & 1485.19 \\
\hline 0.6825 & 1533.05 & 1542.59 & 1538.90 & 1542.28 & 1540.63 & 1492.63 \\
\hline 0.7865 & 1543.84 & 1553.60 & 1550.73 & 1553.36 & 1552.09 & 1500.29 \\
\hline 0.8924 & 1559.31 & 1564.67 & 1563.01 & 1564.53 & 1563.80 & 1508.17 \\
\hline 1.0000 & 1575.78 & 1575.78 & 1575.78 & 1575.78 & 1575.78 & 1516.28 \\
\hline \multicolumn{7}{|c|}{$313.15 \mathrm{~K}$} \\
\hline 0.0000 & 1452.11 & 1452.11 & 1452.11 & 1452.11 & 1452.11 & 1433.59 \\
\hline 0.0929 & 1460.89 & 1462.48 & 1461.11 & 1462.35 & 1461.72 & 1439.69 \\
\hline 0.1872 & 1469.26 & 1472.89 & 1470.41 & 1472.66 & 1471.53 & 1445.96 \\
\hline 0.2831 & 1478.21 & 1483.35 & 1480.03 & 1483.04 & 1481.54 & 1452.39 \\
\hline 0.3805 & 1489.42 & 1493.85 & 1489.99 & 1493.50 & 1491.75 & 1459.01 \\
\hline 0.4795 & 1495.57 & 1504.40 & 1500.29 & 1504.03 & 1502.19 & 1465.81 \\
\hline 0.5802 & 1508.36 & 1515.00 & 1510.97 & 1514.64 & 1512.85 & 1472.80 \\
\hline 0.6825 & 1517.36 & 1525.64 & 1522.04 & 1525.32 & 1523.73 & 1479.99 \\
\hline 0.7865 & 1527.84 & 1536.33 & 1533.52 & 1536.08 & 1534.86 & 1487.38 \\
\hline 0.8924 & 1543.47 & 1547.06 & 1545.45 & 1546.92 & 1546.22 & 1494.98 \\
\hline 1.0000 & 1557.84 & 1557.84 & 1557.84 & 1557.84 & 1557.84 & 1502.80 \\
\hline \multicolumn{7}{|c|}{$318.15 \mathrm{~K}$} \\
\hline 0.0000 & 1437.06 & 1437.06 & 1437.06 & 1437.06 & 1437.06 & 1422.13 \\
\hline 0.0929 & 1446.31 & 1447.28 & 1445.91 & 1447.10 & 1446.51 & 1428.35 \\
\hline 0.1872 & 1455.31 & 1457.54 & 1455.06 & 1457.22 & 1456.16 & 1434.73 \\
\hline 0.2831 & 1464.73 & 1467.84 & 1464.52 & 1467.42 & 1466.00 & 1441.27 \\
\hline 0.3805 & 1475.89 & 1478.18 & 1474.31 & 1477.70 & 1476.06 & 1447.98 \\
\hline 0.4795 & 1481.52 & 1488.56 & 1484.45 & 1488.05 & 1486.32 & 1454.88 \\
\hline 0.5802 & 1494.21 & 1498.98 & 1494.95 & 1498.49 & 1496.80 & 1461.95 \\
\hline 0.6825 & 1502.45 & 1509.44 & 1505.83 & 1509.01 & 1507.51 & 1469.22 \\
\hline 0.7865 & 1512.84 & 1519.94 & 1517.13 & 1519.60 & 1518.45 & 1476.68 \\
\hline 0.8924 & 1527.90 & 1530.47 & 1528.86 & 1530.28 & 1529.62 & 1484.34 \\
\hline 1.0000 & 1541.05 & 1541.05 & 1541.05 & 1541.05 & 1541.05 & 1492.21 \\
\hline
\end{tabular}


Table 2. Percentage deviation between experimental and theoretical values of velocities in o-anisidine + o-cresol system at varying temperatures

\begin{tabular}{|c|c|c|c|c|c|c|c|c|c|c|}
\hline $\mathrm{X}_{1}$ & $\% \mathrm{U}_{\mathrm{N}}$ & $\% \mathrm{U}_{\text {imx }}$ & $\% \mathrm{U}_{\mathrm{Im}}$ & $\% \mathrm{U}_{\mathrm{R}}$ & $\% \mathrm{U}_{\mathrm{J}}$ & $\% \mathrm{U}_{\mathrm{N}}$ & $\% \mathrm{U}_{\mathrm{imx}}$ & $\% \mathrm{U}_{\mathrm{Im}}$ & $\% \mathrm{U}_{\mathrm{R}}$ & $\% \mathrm{U}_{\mathrm{J}}$ \\
\hline & \multicolumn{5}{|c|}{$303.15 \mathrm{~K}$} & \multicolumn{5}{|c|}{$308.15 \mathrm{~K}$} \\
\hline 0.0000 & 000 & 0000 & 0.0000 & 0000 & 7623 & 000 & 0.0000 & 0.0000 & 0.0000 & -1.5149 \\
\hline 0.0929 & 2799 & 1845 & 0.2736 & 271 & 7816 & 2031 & 0.1083 & 0.1946 & 0.1504 & 1.6004 \\
\hline 0.1872 & 4899 & 0.3177 & 0.4785 & 0.3952 & -1.8577 & .3965 & 0.2256 & 0.3814 & 0.3021 & -1.6837 \\
\hline 0.2831 & 5963 & 0.3672 & 0.5813 & 0.4712 & -2.0226 & 0.4948 & 0.2673 & 0.4749 & 0.3700 & -1.8483 \\
\hline 0.3805 & 5412 & 0.2760 & 0.5240 & 0.3975 & -2.3314 & 0.4511 & 0.1878 & 0.4284 & 0.3078 & -2.1385 \\
\hline 0.4795 & 8858 & 0.6048 & 0.8678 & 0.7347 & -2.2390 & 0.7513 & 0.4725 & 0.7275 & 0.6008 & -2.0812 \\
\hline 0.5802 & 5601 & 0.3872 & 0.6428 & 145 & 857 & 764 & 0.3055 & 0.5536 & 0.4313 & 2.4725 \\
\hline 0.6825 & 127 & 4703 & 0.6975 & 0.5845 & 490 & 18 & 0.3812 & 0.6018 & 0.4941 & 365 \\
\hline 0.7865 & 49 & 0.4976 & 34 & 67 & 56 & 23 & 0.4462 & 0.6170 & 0.5343 & 207 \\
\hline 924 & 374 & 0.3307 & 0.4309 & 0.3820 & -3.4987 & 0.3435 & 0.2375 & 0.3349 & 0.2882 & -3.2795 \\
\hline \multirow[t]{2}{*}{1.0000} & 000 & 0.0000 & 0.0000 & 0.0000 & -4.0886 & .0000 & 0.0000 & 0.0000 & 0.0000 & -3.7758 \\
\hline & \multicolumn{5}{|c|}{$313.15 \mathrm{~K}$} & \multicolumn{5}{|c|}{$318.15 \mathrm{~K}$} \\
\hline 000 & 000 & 0.0000 & 0.0000 & 0.0000 & 2752 & 000 & 0. & 0.0000 & 0.0000 & -1.0386 \\
\hline 0.0929 & 0.1086 & 0.0152 & 0.0998 & 0.0568 & -1.4512 & 0.0671 & 0.0275 & 0.0548 & 0.0139 & -1.2419 \\
\hline 0.1872 & 0.2469 & 0.0786 & 0.2312 & 0.1542 & -1.5862 & 0.1534 & 0.0171 & 0.1315 & 0.0583 & -1.4144 \\
\hline 0.2831 & 0.3475 & 0.1234 & 0.3268 & 0.2250 & -1.7465 & 0.2126 & 0.0142 & 0.1838 & 0.0870 & -1.6017 \\
\hline 0.3805 & 0.2974 & 0.0381 & 0.2738 & 0.1568 & -2.0417 & 0.1554 & 0.1070 & 0.1225 & 0.0112 & -1.8907 \\
\hline 0.4795 & 0.5904 & 0.3157 & 0.5656 & 0.4426 & -1.9898 & 0.4754 & 0.1975 & 0.4410 & 0.3239 & -1.7983 \\
\hline 0.5802 & 0.4399 & 0.1729 & 0.4161 & 0.2974 & -2.3573 & 0.3194 & 0.0493 & 0.2864 & 0.1733 & -2.1588 \\
\hline 0.6825 & 0.5455 & 0.3082 & 0.5247 & 0.4200 & -2.4628 & 0.4652 & 0.2252 & 0.4363 & 0.3366 & -2.2119 \\
\hline 0.7865 & 0.5554 & 0.3719 & 0.5395 & 0.4591 & -2.6481 & 0.4691 & 0.2836 & 0.4471 & 0.3705 & -2.3905 \\
\hline 0.8924 & 0.2326 & 0.1281 & 0.2236 & 0.1783 & -3.1415 & 0.1684 & 0.0628 & 0.1560 & 0.1128 & -2.8513 \\
\hline 1.0000 & 0.0000 & 0.0000 & 0.0000 & 0.0000 & -3.5330 & 0.0000 & 0.0000 & 0.0000 & 0.0000 & -3.1696 \\
\hline
\end{tabular}

\section{CONCLUSIONS}

Theoretical evaluations of ultrasonic velocities in binary liquid mixtures are determined, and the validity of different theories is checked. It is observed that out of all the theories Van Dael ideal mixing relation gives best results followed by Rao's theory.

\section{REFERENCES}

[1] Anwar Ali A., Anil Kumar Nain, Soghra Hyder, J. Pure Appl. Ultrasonics 23 (2001) 73.

[2] Narendra K., Narayanamurthy P., Srinivasu Ch., International Journal of Computational Mathematical Ideas 2 (2010) 55-59.

[3] Rao G. V. R., Sarma A. V., Krishna J. S. R., Rambabu C., Indian J. Pure Appl. Phys. 43 (2005) 345-354. 
[4] Madhu R., Aashees A., Manisha G., Shukla J. P., Indian J. Pure Appl. Phys. 40 (2002) 256-263.

[5] Nomoto O., J. Phys. Soc. Japan 13 (1958) 1528-1532.

[6] Subhash C. Bhatia, Rachana Bhatia, Gyan P. Dubey, J. Mol. Liq. 152 (2010) 39-52.

[7] Baluja S., Parsania P. H., Asian J. Chem. 7 (1995) 417-423.

[8] Sravan kumar D., Krishna Rao D., Indian J. Pure Appl. Phys. 45 (2007) 210-220.

[9] Azhagiri S., Jayakumar S., Padmanaban R., Gunasekaran S., Srinivasan S., J. Sol. Chem. 38 (2009) 441-448.

[10] Kumar S., Jeevanandham P., J. Mol. Liquids. 174 (2012) 34-41.

[11] Lide D.R, $76^{\text {th }}$ Edn. 1995, CRC Handbook of Chemistry and Physics. CRC Press, New York.

[12] Anuradha S., Prema S., Rajagopal K., J Pure Appl. Ultrason. 27 (2005) 49.

[13] Saravanakumar K., Baskaran R., Kubendran T. R., J. Appl. Sci. 10 (2010) 1616. 